\title{
Examination of presence and location of the accessory mental foramen, and its implications on the mental nerve block
}

\author{
Fatma Sevmez (D), İlhan Bahşi (D), Mustafa Orhan (D) \\ Department of Anatomy, Faculty of Medicine, Gaziantep University, Gaziantep, Turkey
}

\begin{abstract}
Objectives: It is clinically essential to know the location of accessory mental foramen in the mental nerve anesthesia. The aim of this study was to determine the frequency of accessory mental foramen and examining its morphometric properties.

Methods: A total of 35 adult mandibles of unknown age, gender, and ethnicity were examined. The presence of accessory mental foramen of the mandible was investigated bilaterally. In cases with the accessory mental foramen, its localization, number, and distance relative to the mental foramen were evaluated.
\end{abstract}

Results: Eleven (15.71\%) accessory mental foramens were detected in the 35 mandibles (70 sides) examined. Six (54.55\%) of the accessory mental foramens were on the left side, and 5 (45.45\%) were on the right side.

Conclusion: Knowing the frequency and localization of the accessory mental foramen will make the mental nerve block more effective and facilitate surgical procedures. It should be kept in mind that the presence of accessory mental foramen should be considered in cases where mental nerve block applications are insufficient.

Keywords: accessory mental foramen; mental foramen; mental nerve block

Anatomy 2021;15(2):127-131 C2021 Turkish Society of Anatomy and Clinical Anatomy (TSACA)

\section{Introduction}

The primary goal of nerve blocks and any surgical interventions should be not damaging the critical anatomical structures in the area where the procedure is performed. ${ }^{[1]}$ Particularly, in the last decades, increase demand for dental implantations, cosmetic and orthognathic surgeries had also led to an increase in interest regarding the variations of the maxilla and mandible..$^{[2-4]}$ The mandibular canal, which is one of the critical anatomical structures in the mandible, starts from the mandibular foramen and ends at the mental foramen. ${ }^{[2,5]}$ The inferior alveolar nerve and the corresponding vessels runs in the mandibular canal. Then, this nerve emerges as the mental nerve through the mental foramen. ${ }^{[3,6]}$ After passing through the mental foramen, the mental nerve innervates part of the lower lip, the buccal vestibule, and the gingival mesial side of the first mandibular molar tooth. To prevent damage to the neu- rovascular bundle in this area, the location of the mental foramen must be definitively identified before surgical procedures such as periodontal, periapical, and implant surgeries. ${ }^{[7,8]}$ Besides, the position of the roots of premolar and molar teeth in respect to mental foramen should also be determined before dental treatment. ${ }^{[3,8]}$ The mental foramen is also very important in implant placement in the foraminal region of the mandibular arch. ${ }^{[0]}$ Although the mental foramen is a bilateral opening localized on the anterior surface of the mandible, ${ }^{[3]}$ the presence of accessory mental foramen, ${ }^{[6,10]}$ unilateral absence ${ }^{[11]}$ bilateral absence ${ }^{[12]}$ and hypoplasia of mental foramen ${ }^{[13]}$ have also been reported in the literature. In these variations, the mental nerve or some of its branches exit the mandible through alternative openings. ${ }^{[7]}$

Other foramens located outside the mental foramen on the anterior outer surface of the mandible is called accessory mental foramen. ${ }^{[14]}$ In this case, while the men- 
tal nerve passes through the mental foramen, the accessory mental nerve pass through the accessory mental foramen. ${ }^{\left[{ }^{6]}\right.}$ In the presence of accessory mental foramen, the areas to be innervated by mental and accessory mental nerves will be different, as some of the fibers of the mental nerve will come out of this foramen. In this instance, invasive procedures around the mental foramen may fail, or the mental nerve block may be insufficient. Therefore, it is clinically essential to know the accessory mental foramen.

The aim of this study was to contribute to the literature by determining the frequency of accessory mental foramen and examining its morphometric properties.

\section{Materials and Methods}

A total of 35 adult mandibles of unknown age, gender, and ethnicity were examined in the laboratory of the Department of Anatomy, Faculty of Medicine, Gaziantep
University. The presence of accessory mental foramen of the mandible was investigated bilaterally. In cases with the accessory mental foramen, its localization, number, and distance relative to the mental foramen were evaluated. In the mandibles with accessory mental foramen, photographs were taken with a Nikon D500 camera (Nikon Corp., Tokyo, Japan) and $55 \mathrm{~mm}$ lens, in which the mental foramen and accessory mental foramen were clearly seen, in a standard position that can be repeated during examinations and with an equal distribution of light. All measurements were made on the photographs with the ImageJ software. Descriptive analyzes were performed Statistical Package for Social Sciences (SPSS Version 22, Armonk; NY, USA).

\section{Results}

Eleven $(15.71 \%)$ accessory mental foramens were detected in 35 mandibles (70 sides) (Figure 1). Six (54.55\%) of the accessory mental foramens were on the left side, and 5 of

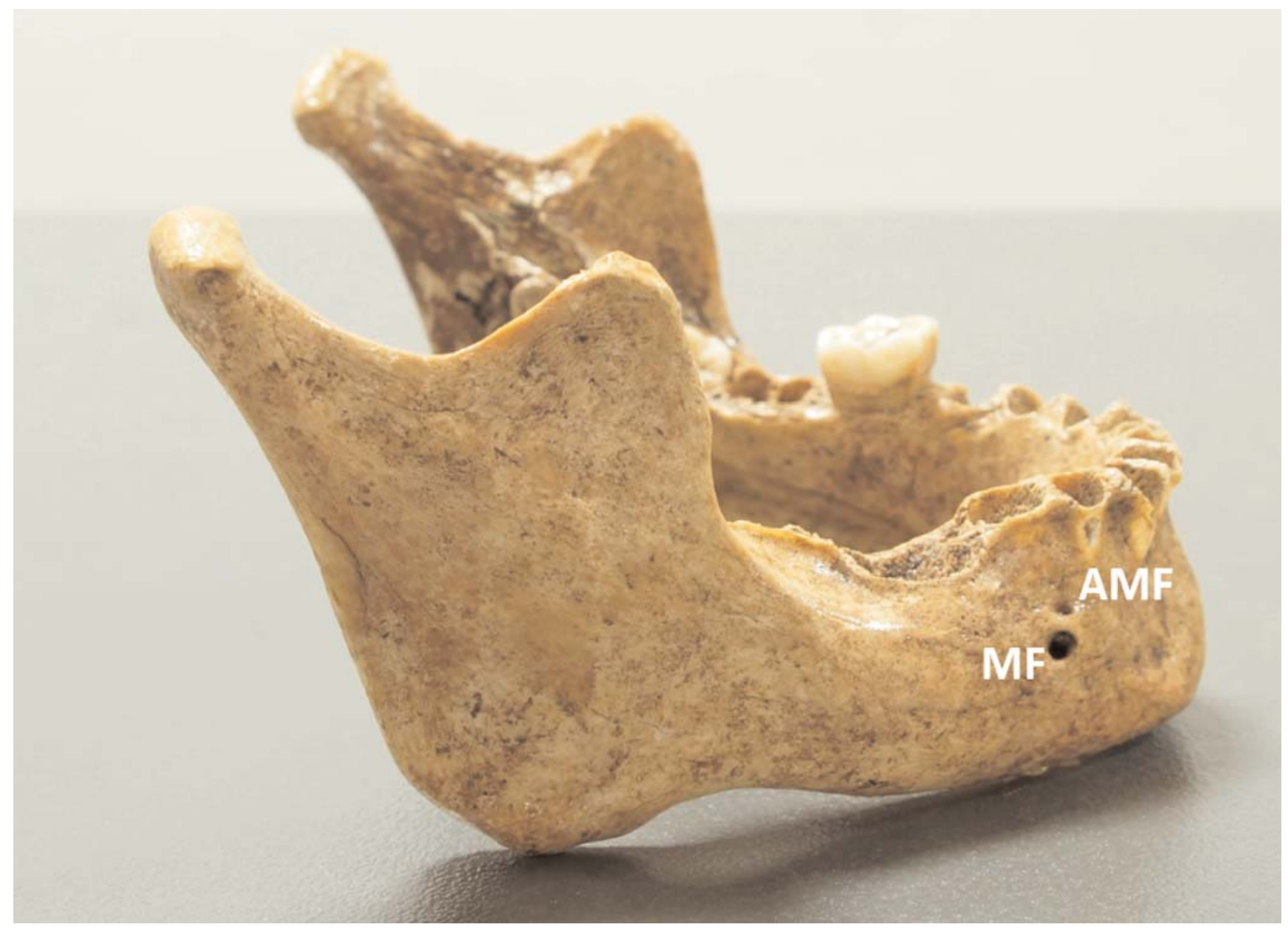

Figure 1. The mental foramen (MF) and accessory mental foramen (AMF) in a mandible. 
them $(45.45 \%)$ were on the right. All accessory mental foramens $(100 \%)$ were unilateral. The distance between the accessory mental foramen and mental foramen varied between 4-12 $\mathrm{mm}$. For the right and left sides, the localization and distance of the accessory mental foramen relative to the mental foramen are shown in Figure 2.

\section{Discussion}

The mental foramen is of great importance for diagnosis, anesthesia, and surgical procedures. ${ }^{[15]}$ Although the mental foramen is usually found as a single foramen on each side of the mandible, there may be more than one foramen in this area. ${ }^{[14]}$ The presence of accessory mental foramen is significant, especially in dentistry ${ }^{[16]}$ Although the accessory mental foramen is a rare structure, it should be well known not to damage the neurovascular bundle during several surgical procedures and prevent inadequate nerve block. ${ }^{[7,17]}$

There are studies examining this variation with panoramic radiography, ${ }^{[18]} \mathrm{CBCT}^{[8,16]}$ and on dry mandibles in the literature. ${ }^{[19]}$ Rahpeyma and Khajehahmadi ${ }^{[4]}$ suggested that the difference in the frequency of its presence is related with the assessment method. Imada et al. ${ }^{[10]}$ reported that this formation was more hardly visible on panoramic graphs. On the other hand, Katakami et al. ${ }^{[20]}$ reported that cone-beam computed tomography scanning is better to detect an accessory mental foramen. Although it is known that the cone-beam computed tomography images clearly show bony structures, ${ }^{[21,2]}$ we think that small structures such as accessory mental foramen is readily seen on bony specimens. However, the most crucial disadvantage of dry bone studies is the lack of information such as gender, age, and ethnicity. ${ }^{[23]}$

There are differences in the literature regarding the localization of the accessory mental foramen in respect to the mental foramen. ${ }^{[17,20,24]}$ Katakami et al..$^{[20]}$ and OliveiraSantos et al. ${ }^{[24]}$ reported that the accessory mental foramen is mostly located lateral to the mental foramen. On the other hand, Neves et al. ${ }^{[17]}$ reported that it was most commonly located inferolateral of the mental foramen. In this study, it was observed that there was a difference between both sides, and it was usually located medially on the right side and laterally on the left side. However, we think that it is difficult to provide statistically significant information because the frequency of this variation is low. OliveiraSantos et al ${ }^{[24]}$ reported that the longest distances between the mental and accessory mental foramens were $7.4 \mathrm{~mm}$ horizontally, and $3.58 \mathrm{~mm}$ vertically. However, in this
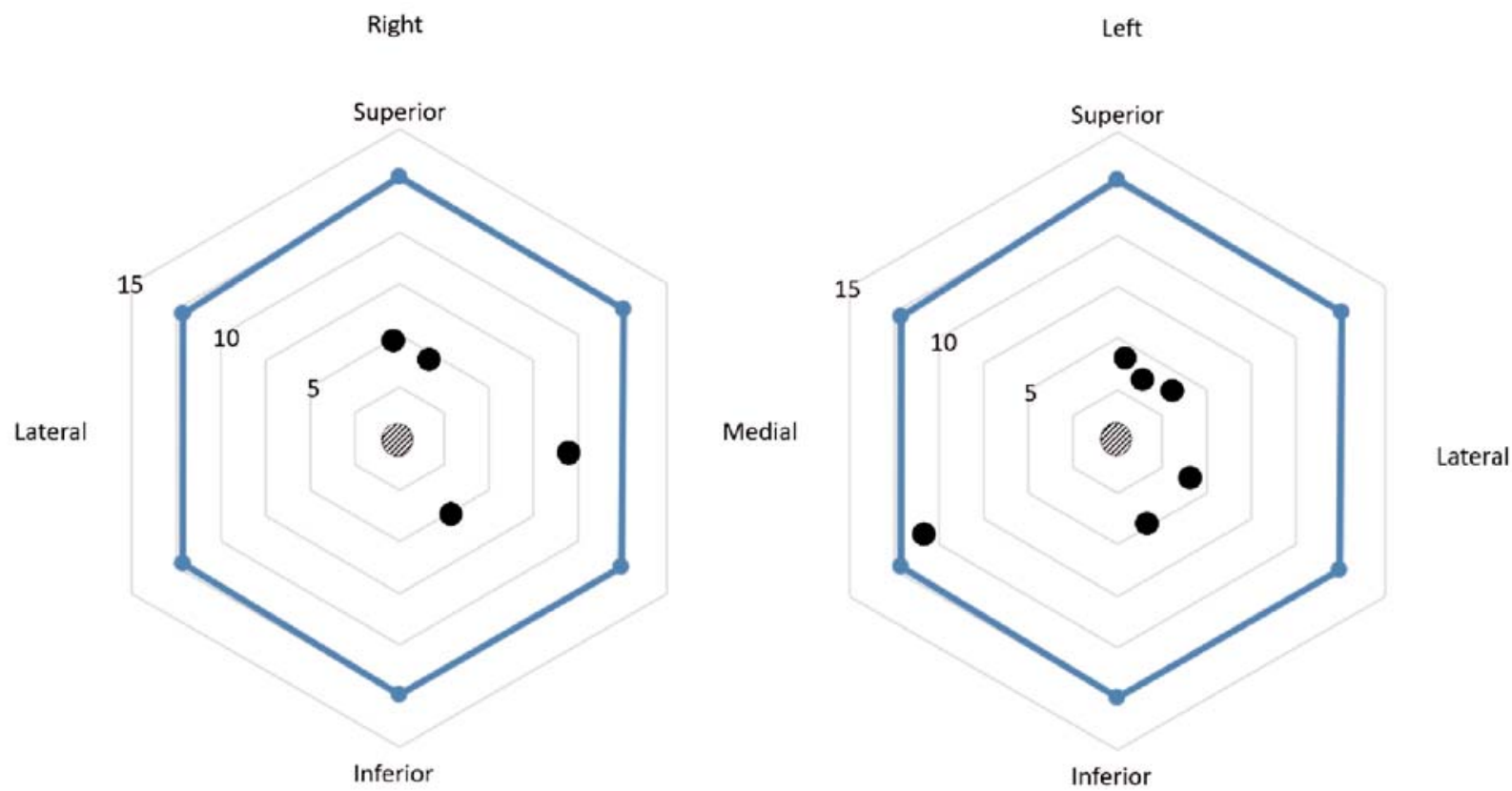

Mental Foramen

Accessory Mental Foramen

Figure 2. Localization of the accessory mental foramens according to the mental foramen (the unit for values 5,10 and 15 is $\mathrm{mm}$ ). 
study, the longest distance was found to be $12 \mathrm{~mm}$. We suggest that as this distance increases, the failure rate in the mental nerve block may also increase. Therefore, we think that this distance worth consideration in addition to the presence of the accessory mental foramen.

\section{Conclusion}

Although the mental foramen is usually found as a single foramen on each side of the mandible, an accessory mental foramen can also be found with a low but significant incidence. For this reason, knowing the frequency and localization of the accessory mental foramen will increase the effectivity of the nerve blocks, facilitate surgical procedures involving the lower jaw and minimize the damage to the vascular-nerve structures in this area. In addition, the presence of accessory mental foramen should be considered in cases where mental nerve block applications are insufficient.

\section{Acknowledgments}

The authors would like to express their sincere gratitude to the donors and their families.

\section{Conflict of Interest}

The authors declare that they do not have any conflict of interest.

\section{Author Contributions}

FS: protocol/project development, data collection or management, data analysis and manuscript writing/editing; İB: protocol/project development, data collection or management, data analysis and manuscript writing/editing; MO: protocol/project development, data collection or management, data analysis and manuscript writing/editing.

\section{Ethics Approval}

The study was conducted in accordance with the ethical rules of the Declaration of Helsinki and its later amendments. Scientific studies on dry bones in our institution do not require ethical approval.

\section{Funding}

None.

\section{References}

1. Bahsi I, Orhan M, Kervancioglu P, Yalcin ED. Morphometric evaluation and clinical implications of the greater palatine foramen, greater palatine canal and pterygopalatine fossa on CBCT images and review of literature. Surg Radiol Anat 2019;41:551-67.

2. Xiao L, Pang W, Bi H, Han X. Cone beam CT-based measurement of the accessory mental foramina in the Chinese Han population. Experimental and Therapeutic Medicine 2020;20:1907-16.
3. Caglayan F, Sumbullu MA, Akgul HM, Altun O. Morphometric and morphologic evaluation of the mental foramen in relation to age and sex: an anatomic cone beam computed tomography study. J Craniofac Surg 2014;25:2227-30.

4. Rahpeyma A, Khajehahmadi S. Accessory mental foramen and maxillofacial surgery. J Craniofac Surg 2018;29:e216-7.

5. Sevmez F, Orhan M, Bahsi I, Yalcin ED. Examination of the safe zone in mandibular ramus osteotomies. J Craniofac Surg 2021;32:2219-22.

6. Orhan AI, Orhan K, Aksoy S, Ozgul O, Horasan S, Arslan A, Kocyigit D. Evaluation of perimandibular neurovascularization with accessory mental foramina using cone-beam computed tomography in children. J Craniofac Surg 2013;24:e365-9.

7. Torres MG, Valverde Lde F, Vidal MT, Crusoe-Rebello IM. Accessory mental foramen: a rare anatomical variation detected by cone-beam computed tomography. Imaging Sci Dent 2015;45:61-5.

8. Kalender A, Orhan K, Aksoy U. Evaluation of the mental foramen and accessory mental foramen in Turkish patients using cone-beam computed tomography images reconstructed from a volumetric rendering program. Clin Anat 2012;25:584-92.

9. Greenstein G, Tarnow D. The mental foramen and nerve: clinical and anatomical factors related to dental implant placement: a literature review. J Periodontol 2006;77:1933-43.

10. Imada TS, Fernandes LM, Centurion BS, de Oliveira-Santos C, Honorio HM, Rubira-Bullen IR. Accessory mental foramina: prevalence, position and diameter assessed by cone-beam computed tomography and digital panoramic radiographs. Clin Oral Implants Res 2014;25:e94-9.

11. Ulu M, Tarim Ertas E, Gunhan F, Yircali Atici M, Akcay H. Unilateral absence of mental foramen with surgical exploration in a living human subject. Case Rep Dent 2016;1971925.

12. Matsumoto K, Araki M, Honda K. Bilateral absence of the mental foramen detected by cone-beam computed tomography. Oral Radiology 2012;29:198-201.

13. da Silva Ramos Fernandes LM, Capelozza AL, Rubira-Bullen IR. Absence and hypoplasia of the mental foramen detected in CBCT images: a case report. Surg Radiol Anat 2011;33:731-4.

14. Simonton FV. Mental foramen in the anthropoids and in man. Am J Phys Anthropol 1923;6:413-21.

15. von Arx T, Lozanoff S, Bosshardt D. Accessory mental foramina: anatomy and histology of neurovascularisation in four cases with apical surgery. Oral Surgery 2014;7:216-27.

16. Lam M, Koong C, Kruger E, Tennant M. Prevalence of accessory mental foramina: a study of 4,000 CBCT scans. Clin Anat 2019;32: $1048-52$.

17. Neves FS, Nascimento MC, Oliveira ML, Almeida SM, Boscolo FN. Comparative analysis of mandibular anatomical variations between panoramic radiography and cone beam computed tomography. Oral Maxillofac Surg 2014;18:419-24.

18. Capote TS, Goncalves Mde A, Campos JA. Retromolar canal associated with age, side, sex, bifid mandibular canal, and accessory mental foramen in panoramic radiographs of Brazilians. Anat Res Int 2015; 434083.

19. Singh R. Evaluation of position, shape, size and incidence of mental foramen and accessory mental foramen in Indian adult human skulls. Anatomy 2011;5:23-9.

20. Katakami K, Mishima A, Shiozaki K, Shimoda S, Hamada Y, Kobayashi K. Characteristics of accessory mental foramina observed on limited cone-beam computed tomography images. J Endod 2008; 34:1441-5. 
21. Ucar H, Bahsi I, Orhan M, Yalcin ED. The radiological evaluation of the crista galli and its clinical implications for anterior skull base surgery. J Craniofac Surg 2021;32:1928-30.

22. Bahsi I, Orhan M, Kervancioglu P, Yalcin ED, Aktan AM. Anatomical evaluation of nasopalatine canal on cone beam computed tomography images. Folia Morphol (Warsz) 2019;78: 153-62.

ORCID ID:

F. Sevmez 0000-0002-3110-2881;

I. Bahşi 0000-0001-8078-7074,

M. Orhan 0000-0003-4403-5718

deomed。
23. Bahsi I. An anatomic study of the supratrochlear foramen of the humerus and review of the literature. European Journal of Therapeutics 2019;25:295-303.

24. Oliveira-Santos C, Souza PH, De Azambuja Berti-Couto S, Stinkens L, Moyaert K, Van Assche N, Jacobs R. Characterisation of additional mental foramina through cone beam computed tomography. J Oral Rehabil 2011;38:595-600.

Correspondence to: Ilhan Bahşi, MD, PhD Department of Anatomy, Faculty of Medicine, Gaziantep University, Gaziantep, Turkey Phone: +90 3423606060 / 4655 e-mail: dr.ilhanbahsi@gmail.com

Conflict of interest statement: No conflicts declared.

This is an open access article distributed under the terms of the Creative Commons Attribution-NonCommercial-NoDerivs 4.0 Unported (CC BY-NCND4.0) Licence (http://creativecommons.org/licenses/by-nc-nd/4.0/) which permits unrestricted noncommercial use, distribution, and reproduction in any medium, provided the original work is properly cited. How to cite this article: Sevmez F, Bahşi I, Orhan M. Examination of presence and location of the accessory mental foramen, and its implications on the mental nerve block. Anatomy 2021;15(2):127-131. 